\title{
Potential Adverse Effect of Ultrasound on the Electroretinogram of Retina and the Protective Role of Different Types of Antioxidants
}

\author{
Ayman Ahmed ${ }^{1}$, Mervat Ahmed Ali ${ }^{2,}$, Mona Mostafa Gamal ${ }^{2}$, Abdel Sattar Mohamed Sallam ${ }^{1}$, \\ Elsayed Mahmoud Elsayed ${ }^{1}$
}

${ }^{1}$ Collage of Science, Ain Shams University, Cairo, Egypt

${ }^{2}$ Research Institute of Ophthalmology, Giza, Egypt

Email address:

Mervat_galal18@yahoo.com(M. A. Ali)

${ }^{*}$ Corresponding author

\section{To cite this article:}

Ayman Ahmed, Mervat Ahmed Ali, Mona M. Gamal, Abdel Sattar Mohamed Sallam, Elsayed Mahmoud Elsayed. Potential Adverse Effect of Ultrasound on the Electroretinogram of Retina and the Protective Role of Different Types of Antioxidants. European Journal of Biophysics. Vol. 8, No. 2, 2020, pp. 20-25. doi: 10.11648/j.ejb.20200802.12

Received: August 19, 2020; Accepted: September 5, 2020; Published: September 24, 2020

\begin{abstract}
Background: Applications of ultrasound in medicine for therapeutic purposes have been an accepted and beneficial use of ultrasonic biological effects for many years but exposure to ultrasound can generate oxidative stress. Aim: The aim of the present study is to investigate the changes in the function of the retina due to ultrasound exposure and the protecting role of vitamin $C$ and/or $\beta$-carotene by using electroretinogram (ERG). Materials and Methods: Newzealand albino rabbits of both sexes classified into five groups. Group (I) used as control group. The eyes of group (II) Insonificated with continuous ultrasound waves $\left(1.5 \mathrm{~W} / \mathrm{cm}^{2}\right.$ at $2.8 \mathrm{MHz}$ ) for 20,40 and 60 minutes. Group (III) was insonificated with vitamin C instillation and group (IV) insonificated after $\beta$-carotene supplementation. Group (V) was insonificated after combination of the two treatments. Results: Statistically significant reduction $(\mathrm{P}<0.05)$ in a- waves amplitude after insonification and exceeding with increase exposure time 20, 40 and $60 \mathrm{~min}$ was observed. The amplitudes of a-wave after treatment with topical and supplemented or both remained significantly larger compared with those exposure to US only but its latency lower than that found in insonified groups. In addition, the b-waves were found to follow the same behavior as a-wave. Conclusion: The study recommended considering oral $\beta$-carotene antioxidant in combination with vitamin $C$ eye drops as a medical tool and personal protective in ultrasound equipment in provide safety.
\end{abstract}

Keywords: Ultrasound, Vitamin C, $\beta$-carotene, Electroretinogram, Retina, Rabbit

\section{Introduction}

Ultrasound (US) Applications in medicine have long been an established and beneficial use of ultrasonic biological effects for therapeutic purposes. Low power ultrasound with a frequency of around $1 \mathrm{MHz}$ has been widely commonly used for physical therapy in conditions such as tendinitis or bursitis since the $1950 \mathrm{~s}$. Ultrasound energy Ultrasound energy has significant mechanical, biochemical, thermal and cellular effects. Concern over ultrasound protection led many agencies to devise regulatory restrictions on the machine performance intensities. During ultrasound imaging The visual display of thermal and mechanical indices provides an assist to restrict the system performance.

Rwei et al., [1] stated that US exposure could generate oxidative stress that is comparable to traditional $\mathrm{H}_{2} \mathrm{O}_{2}$ administrations. The effect of generating oxidative stress is adjustable by means of ultrasound parameters, including the pulsed or continuous wave, US intensity and microbubbles addition.

Chen et al., [2] found that intense-focused ultrasound (IFUS) rejuvenation may result in heat injury, leading to an acute increase in intraocular pressure (IOP). Heat damage to zonular fibers can cause spasm and myopia in accommodation. 
Carotenoids are naturally occurring pigments found in most fruits and vegetables, plants, algae, and photosynthetic bacteria. Humans cannot synthesize carotenoids and must ingest them in food or via supplementation. Carotenoids have a variety of human health roles. They exert antioxidant effects mainly, but individual carotenoids can also work by other mechanisms; for example, $\beta$-carotene has a function of pro-vitamin A, while lutein/zeaxanthin constitutes macular pigment in the eye. Lutein's role in reducing development of age-related macular eye disease and cataracts is strengthening; an intake recommendation will help generate awareness in the general population to get sufficient intake of lutein rich foods [3]. Li et al., [4] reported that carotenoid supplementation can improve human visual efficiency, but no validated rodent model is yet available to test its effects on visual function in laboratory animals. They showed that mice deficient in $\beta$-carotene oxygenase 2 (BCO2) and/or $\beta$ carotene oxygenase 1 (BCO1) enzymes can accumulate carotenoids in their retinas, allowing them to investigate the effects of carotenoids on the visual performance of mice. The effects of a carotenoid supplementation on retinal thickness and macular function of patients with diabetes was investigated by Moschos et al.,[5] suggesting the use of carotenoid supplements may be of benefit for improving visual function of type 2 diabetes patients. Giannaccare et al.,[6] has been shown cartenoids significantly reduce retinal ganglion cells apoptosis that is responsible for the progression of retinal damage in glaucoma and in other optic neuropathies, as well as RPE cells death that causes age macular degeneration (AMD) development.

$\beta$-carotene $\left(\mathrm{C}_{40} \mathrm{H}_{56}\right)$ present in abundance in the human diet and subsequently found in all human tissues including blood. Due to its high bioactivity, it is also widely used in medicine. Among the various roles of $\beta$-carotene in the human body, the essential one relates to the supply of provitamin A, thus influencing embryonic development, correct growth, and sight. It is considered an inhibitor of some genes, and it has anticancer and antioxidant properties $[7,8]$.

Ascorbic Acid is a water-soluble natural vitamin (Vitamin $\mathrm{C}, \mathrm{C}_{6} \mathrm{H}_{8} \mathrm{O}_{6}$ ). Vitamin $\mathrm{C}$ is an effective antioxidant and reducing agent that acts in fighting bacterial infections, in detoxification reactions, and collagen formation in fibrous tissue, teeth, bones, connective tissue, skin, and capillaries. Vitamin $\mathrm{C}$ present in citrus and other fruits as well as in vegetables, cannot be produced or stored by humans and must be obtained in the diet. It is possible that any ascorbic acid diffuses into the vitreous cavity from the aqueous humor. However, McGahan [9] suggested that Vitamin C might also enter the vitreous humor from other sources; perhaps some passes out of the retina. In most species, the concentration of ascorbic acid in the vitreous humor is the same as that in aqueous humor or slightly lowers [10]. Vitamin $\mathrm{C}$ is concentrated into the neural retina to a similar degree as it is concentrated into the aqueous humor. On this basis, one might expect the retina, in particular the RPE, to have the ability to transport ascorbic acid.
The big challenge facing the pharmacologist and formulation scientist today is the delivery of ocular drugs. The most effective and patient compliant route for drug administration is topical eye drop. Various precorneal, dynamic and static ocular barriers limit the delivery of drugs to the targeted ocular tissues. The therapeutic drug levels are not maintained in target tissues for longer period. Over the past two decades, research into ocular drug delivery accelerated advanced towards developing a new, safe and patient compliant formulation and drug delivery [11,12].

In the present work, we study the recorded a and b- waves of rabbit ERG and their variations due to the exposure to US with different duration $(20,40$ and $60 \mathrm{~min})$ at frequency $2.8 \mathrm{MHz}$ and intensity $1.5 \mathrm{~W} / \mathrm{cm}$. In addition, the protective role of topical Vitamin $\mathrm{C}$ (vit $\mathrm{C}$ ) and $\beta$-carotene supplementation due to US were investigated.

\section{Materials and Methods}

\subsection{Experimental Animals}

Sixty-five healthy mature Newzealand rabbits of both sexes, weighing 2-2.5 Kg were used in this study. The rabbits were selected from the animal house at Research Institute of Ophthalmology, Giza, Egypt. The experimental protocol approved by the local ethical committee that applies ARVO (The Association for Research in Vision and Ophthalmology) statements for using animals in ophthalmic and vision research. All animals were housed two to three rabbits per cage in a central temperature of $20-25^{\circ} \mathrm{C}$ and fed on a laboratory balanced diet. All procedures were conducted according to the principles enunciated in the guide for care and use of laboratory animals. Rabbits were classified into four groups according to the following:

Group I: containing five rabbits were used as control group.

Group II: Contains 15 rabbits subdivided into three subgroups (5 rabbits and 10 eyes each), the three subgroups were insonificated for 20, 40 and 60 minutes with ultrasound.

Group III: Contains 15 rabbits subdivided into three subgroups ( 5 rabbits and 10 eyes each). As the previous group, the three subgroups were insonificated for 20, 40 and 60 minutes with ultrasound. Animals were topically treated with 1 drop (approximately $50 \mu \mathrm{l}$ ) of the $10 \%$ ascorbic acid [13] was used as eye drops every 15 minutes, starting one hour before insonification, until the end of ultrasound exposure.

Group IV: Contains 15 rabbits supplemented with 15 $\mathrm{mg} / \mathrm{kg} \beta$-Carotene for two weeks [14] then subdivided into three subgroups ( 5 rabbits and 10 eyes each). The eyes of the three subgroups were insonificated for 20, 40 and 60 minutes with ultrasound and recorded ERG after 24 hours of ultrasound exposure.

Group V: Consists of 15 rabbits supplemented with 15 $\mathrm{mg} / \mathrm{kg} \beta$-Carotene for two weeks then subdivided into three subgroups ( 5 rabbits and 10 eyes each). The eyes of the three subgroups were insonificated for 20,40 and $60 \mathrm{~min}$ with ultrasound. Moreover, one hour before insonification, until 
the end of ultrasound exposure $10 \%$ ascorbic acid was used as eye drops every 15 minutes.

\subsection{Ultrasound Insonification}

\subsubsection{Properties of Ultrasonic Transducer}

Ultrasound transducer consists of piezoelectric crystal (Barium zirconate, titanium). Type SVHSP101 and a conical base ended with a fine tip transducer with very low quality factor (Q) to give a very wide nearly flat, frequency/ output power. The transducer converts the pulsating voltage output of the generator into a train of mechanical pulses in the ultrasonic range with a power proportional to the applied voltage. The main intensity output is $1.5 \mathrm{watt} / \mathrm{cm}^{2}$ at about frequency $2.8 \mathrm{MHz}$.

\subsubsection{Rabbit Insonification}

The rabbits were anesthetized by injection with $0.1 \mathrm{ml} / \mathrm{kg}$ separine as muscle relaxant and $50 \mathrm{mg} / \mathrm{kg}$ ketamine hydrochloride was injected intramuscularly after 15 minutes. The rabbits placed in a lateral position on an operating table and their body temperature was maintained at $37^{\circ} \mathrm{C}$. The eyes dilated with one drop of $1 \%$ mydriacyl. After adequate anesthesia was obtained, the eyelid was open with a stainless steel speculum. Insonification of rabbit eyes carried out with continous ultrasound waves $\left(1.5 \mathrm{~W} / \mathrm{cm}^{2}\right.$ at $2.8 \mathrm{MHz}$ by using a focus piezoelectric transducer in a direct contact with the cornea. Good coupling maintained with $10 \%$ viscous phenylpherine. The transducer positioned so that the ultrasonic beam aligned to achieve perpendicular transmission through the cornea [15].

\subsection{Electroretinogram (ERG)}

\subsubsection{Animal Preparation}

After 24 hours of ultrasound insonification, the animals were dark adapted for one hour before the electrophysiological recording. They anesthetized by xylazine $(21 \mathrm{mg} / \mathrm{kg}$ of body weight) as muscle relaxant, and ketamine hydrochloride (45 $\mathrm{mg} / \mathrm{kg}$ ) administrated intramuscularly. Supplementary anesthetic was given as required by the same route during the course of the experiment.

Animals placed on the pad of an operating table where their body temperature maintained at $37^{\circ} \mathrm{C}$. Each rabbit is positioned with its head resting to one side and local anesthetizing eye drops are applied. The pupil of the recorded eye dilated with topical $1 \%$ mydriacyl. Hair removal around the eye area is essential during the preparation period before the undergoing of the experiment.

\subsubsection{Light Stimulation}

A white flash used in this work with fixed intensity (4 lux) and duration $(0.2 \mathrm{~s})$. The distance between the flash and the eye (for control) is changed and the corresponding ERG wave in each case is recorded, it is noticed that the amplitude of the b-wave is increased with the increase of the light intensity and reached saturation at $12 \mathrm{~cm}$ from the source. One more point is that, at this distance no effects from the source are noticed. That is considered the favorable condition for obtaining an ERG suitable for testing effects.

\subsubsection{Electrophysiological Recording}

The electroretinogram is recorded by using sensor PS-2111 and its electrodes (PASCO, Roseville, CA) which connect to PASPORT interface direct to the computer. One electrode is placed at the corneal periphery as active electrode; the other electrode is placed on the skin of the lower eyelid as a reference one. The last electrode is placed on the ear as an earthed one. The result of electrophysiological signals is collected and analyzed by data studio 1.9.8 software (PASCO, Roseville, CA). Before starting the measurements, ERG testing for all rats is carried out to establish baseline standards.

\section{Results}

In this part of experimental work, we studied the flash electroretinogram which is a valuable tool for assessing retinal function The ERG serves to register the potential of retinal cells in response to light. The assessment of retinal electrical activity was conducted with a- and b-wave amplitude of the electroretinogram: a-wave is a negative wave reflecting the functional activity of photoreceptors, and b-wave is a positive wave reflecting the electrical activity of bipolar and Muller cells with the possible involvement of the horizontal and amacrine cells. Figure 1 illustrated the records of ERG to control rabbits group and exposed to U. S. after 20, 40 and $60 \mathrm{~min}$ of insonification compared to treated groups with vit. $\mathrm{C}$ and /or $\beta$-carotene. For the control rabbits, the a-wave amplitude which corresponds to photoreceptor function was on the average of $25 \pm 3 \mu \mathrm{v}$ while the average b-wave which reflects the electrical activity in the inner retina subsequent to photoreceptor stimulation was $88 \pm 5 \mu \mathrm{v}$.

Figures 2 and 3 illustrated a-wave amplitude and duration (latency) respectively as a function of the time of exposure to US 20, 40 and $60 \mathrm{~min}$ using frequency $2.8 \mathrm{MHz}$ at intensity $1.5 \mathrm{w} / \mathrm{cm}$ and after treatment with topical vitamin $\mathrm{C}$ and supplementation of $\beta$-carotene or combination between the two treatments. It is clear from figures that statistically significant reduction in a- waves amplitude and it was apparent, after insonification and exceeding with increase exposure time 20, 40 and 60 , the amplitudes were $11 \pm 1,4 \pm 1$, and $5 \pm 1$ respectively and latency of a-wave increase from $15 \pm 1$ to $27 \pm 2$, $30 \pm 2$ and $34 \pm 1$, respectively. The amplitudes of a-wave after treatment with topical and supplemented or both remained significantly larger compared with those exposure to US only but its latency lower than that found in insonified groups.

The b-waves was found to follow the same behavior as in a- wave as shown in figures 4 and 5 that showed relation between different time of ultrasound exposure (20, 40 and 60min) and amplitudes of b-waves. After topical vitamin $\mathrm{C}$ or supplemented $\beta$ - carotene or both and its latency at same frequency and intensity, antioxidant either oral or topical is considered as an effective in preventing damage due to ultrasound. 


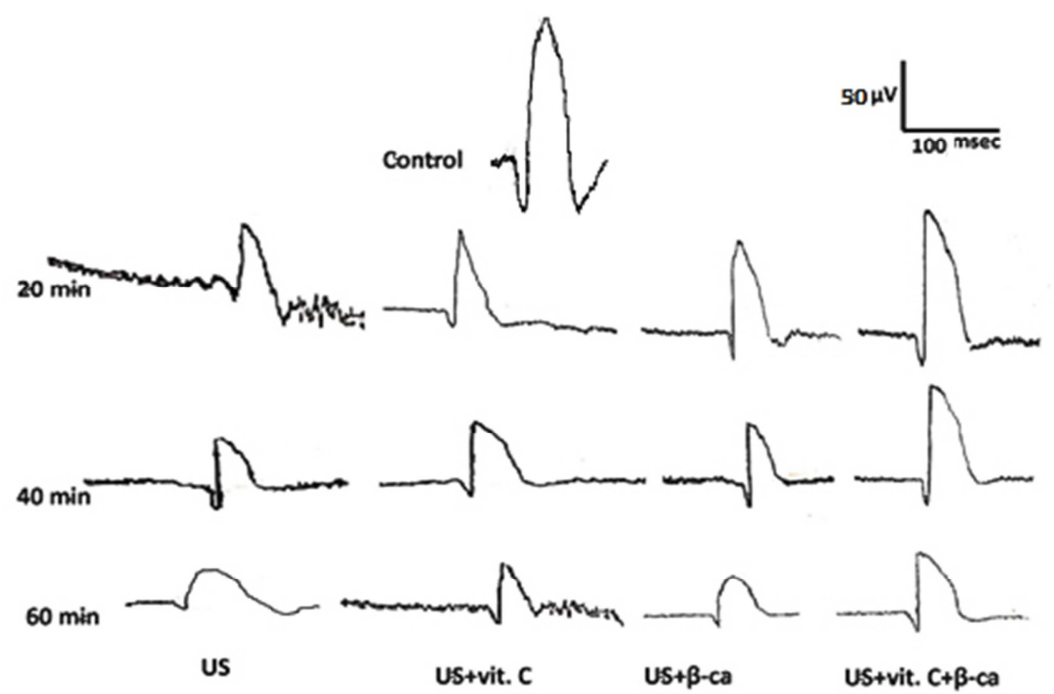

Figure 1. ERG records for control rabbits and those exposed to U. S. for 20, 40 and 60 min compared to treated groups with vit. C and /or $\beta$-carotene.

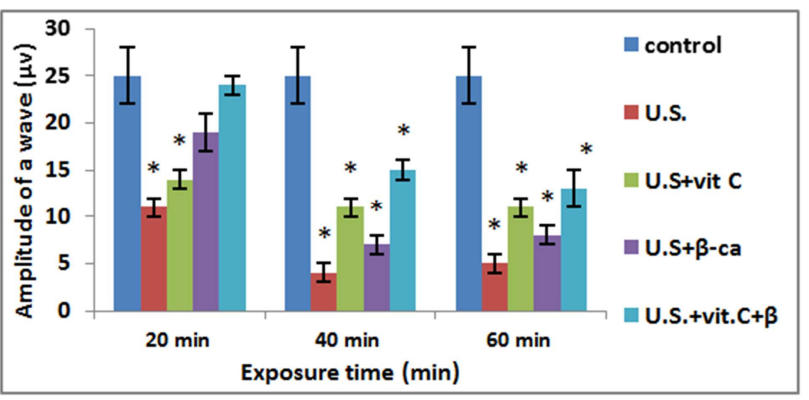

Figure 2. Histogram indicated amplitude of a-waves for all the studied groups compared to control. * Statistical significant $(p<0.05)$.

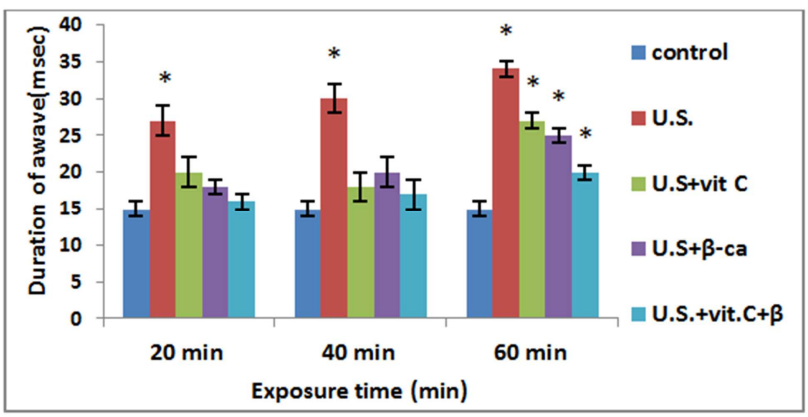

Figure 3. Histogram indicated duration (latency) of a-waves for all the studied groups compared to control. * Statistical significant $(p<0.05)$.

\section{Discussion}

Ultrasound has broad ranging medical applications for biological tissue cutting, ablation or fragmentation, and removal. However, despite its widespread clinical application, there is an incomplete understanding of the mechanism of tissue failure, removal and damage.

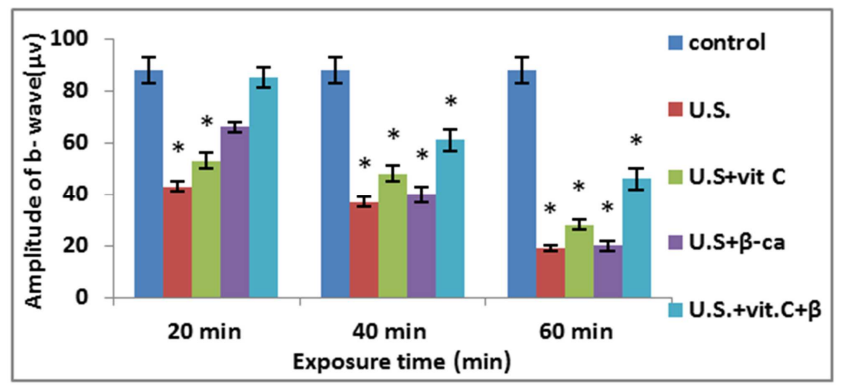

Figure 4. Histogram indicated amplitude of b-waves for all the studied groups compared to control. * Statistical significant $(p<0.05)$.

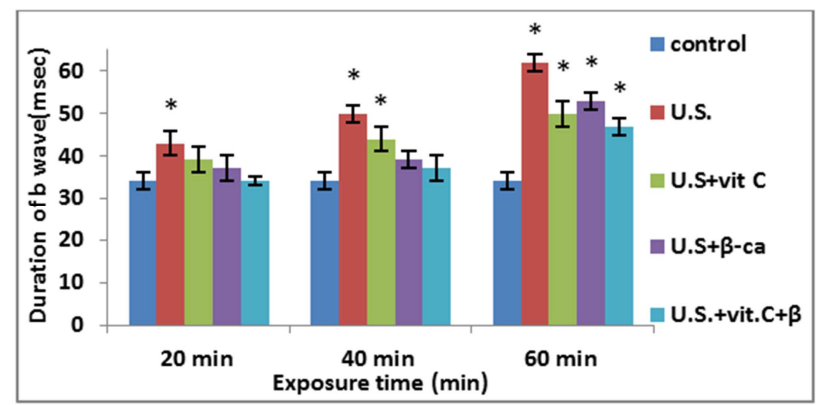

Figure 5. Histogram indicated duration (latency) of b-waves for all the studied groups compared to control. * Statistical significant $(p<0.05)$.

In order to perform quantitative analysis of photoreceptors response to retinal pigment epithelium and neurosensory retinal destruction, ERG done at different ultrasound exposure time. The obtained data of ERG showed changes in the physical parameters of a-and b-waves. These changes depend on intensity and duration of exposure.

The percentage decrease in a- and b-waves after US exposure period 20, 40 and 60 was found to be $56 \%, 84 \%$ and $80 \%$ for a- wave and $51 \%, 58 \%$ and $78 \%$ for b- wave respectively. After 20, 40and 60 minutes of exposure to ultrasound, figures 6 and 7 showed ERG disturbances, a-and 
b-waves amplitudes reduced with increase exposure time.

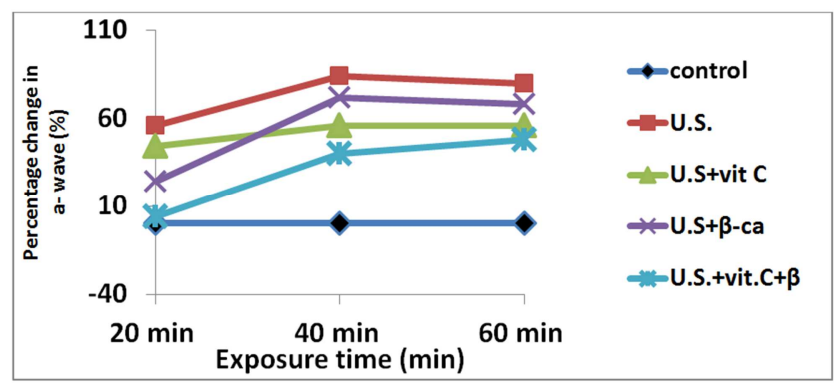

Figure 6. Percentage change of a-wave due to insonification for 20.40 and 60 min compared to control and treated with vitamin $C$ and/or $\beta$-carotene.

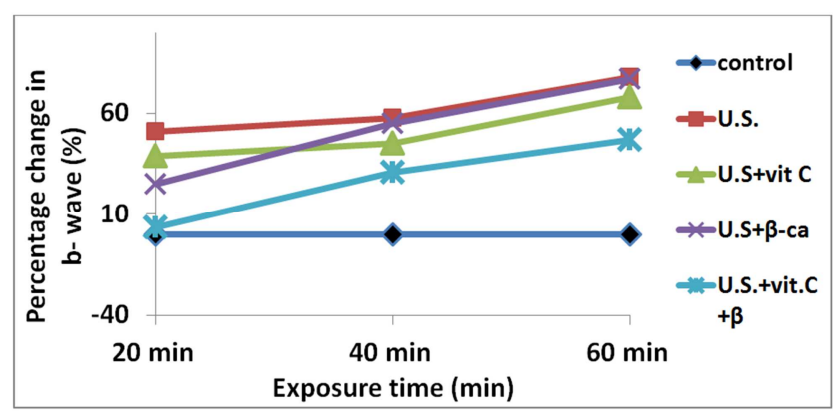

Figure 7. Percentage change of b-wave due to insonification for 20.40 and 60min compared to control and treated with vitamin $C$ and/or $\beta$-carotene.

The reaction of biological tissue to US can be to a certain extent changeable and depends on the acoustic and biological properties as well as on site and role of expose tissue. Ultrasound in liquids leads to "cavitation," a strategy creating micro explosions which may cause mechanical destruction. The chemical effects of ultrasound in aqueous solutions are due to acoustic cavitation, which alludes to the arrangement, expansion, and collapse of small gas bubbles in liquids. The exceptionally high temperatures (several thousand $\mathrm{K}$ ) and pressures (several hundred atmospheres) of collapsing gas bubbles lead to the thermal dissociation of water vapor into. $\mathrm{OH}$ radicals and. $\mathrm{H}$ atoms [16].

The starting tissue harm is watched within the photoreceptors and the outer nuclear layer. It subsequently showed up from a-wave reduction. The potential US harm components happening in tissues incorporate change in global biomechanical properties, histomorphological changes, protein denaturation and tissue necrosis [17]. In addition, all of these retinal changes appeared at the inner retinal layers, these changes explain the reduction in amplitude of b- wave. The increase in latency of a- and bwave would result from greater defect in signal transmission between photoreceptor and bipolar cells.

Concerning oral and topical protection, exogenous nutrients such as vitamin $\mathrm{C}$, topical and oral $\beta$-carotene (a precursor of vitamin A), all contribute to the formation defense against ultrasound damage and act as antioxidants. The injure mechanism is weakly understood and works for harm minimization have not been clearly characterized. For this purpose, we assess in vivo experiments using a topical application containing vitamin $\mathrm{C}$ and oral supplementation with $\beta$-carotene antioxidant.

The results indicated that ultrasound exposure for 20 minutes lead to change the percentage in a- wave to $44 \%, 24 \%$ and $4 \%$ and percentage change in $\mathrm{b}$-wave was 39\%, 25\%and $3.4 \%$ after topical ascorbic acid, oral $\beta$-caroten and both respectively. It is clear that oral $\beta$-caroten antioxidant was more effective at lowest period of exposure and has a lasting effect much longer than topical one. A characteristic, orally administered supplement can relieve eyes from the inside out by motivating healthy tear making to re-establish eyes delicate system. The role of betacarotene as a free radical scavenger has been well documented by many researchers [18-20]. Our findings are largely consistent with those of earlier studies of Tso [14] and Ham and coworkers [21]. They reported that threshold of minimal retinal damage from blue light, was increase by approximately $44 \%$ after supplementation with beta-carotene. In prolonged exposure 40 and 60 minutes the percentage change in a -wave 56\%, $72 \%$ and $40 \%$ after exposure period 40 minutes for topical, oral and both respectively and percentage change of $b$ wave after 60 minutes was $56 \%, 68 \%$ and $48 \%$, respectively.

Our findings illustrate that prolonged US exposure 40 and 60 able to harm retinal integrity in exposed rabbits and that a topical treatment with antioxidant eye drops makes the ultrastructural modifications less dramatic. In fact, the biological structures are exposed to physiological shifts of $3^{\circ} \mathrm{C}$ without permanent injury. Hatab et al., [22] found that if US increased tissue temperature by $4^{\circ} \mathrm{C}$, then injury occurred after 16-minute exposures but, when temperature increased by $6^{\circ} \mathrm{C}$, then changes occurred at 1 minute. One of the most severe complications that may occur during insonification is caused by overheating of the ultrasonic tip; so, irrigating solutions used to dissipate heat and assist removal of debris inadvertently also wash away natural antioxidants [23].

Several researchers have investigated how intraocular irrigation solutions may influence the retina. At the biochemical level, Furthermore, animal studies showed that ascorbic acid applied to the irrigating solution could serve as free-radical scavengers to avoid endothelial cell damage from phacoemulsification [13, 24]. Another suggestion for the protection of topical ascorbic acid was that the cavitationinduced epithelial damage was the main reason for corneal permeability increase [25]. In turn, the extent of the infusion fluid/retina interaction will mediate by vitreous through either diffusion and/or hydration, the topical treatment came into contact with the retina [26]. In prolonged ultrasound exposure, topical vitamin $\mathrm{C}$ can do both dissipate heat and serve as free-radical scavengers to counteract the detrimental effects of US on the retina and minimizing the electrophysiology changes.

\section{Conclusion}

Both topical vitamin $\mathrm{C}$ and oral $\beta$-carotene has a role in protect the retina from ultrasound damage. Oral $\beta$-carotene antioxidant was more effective and has a lasting effect in lower exposure duration 20 minutes but by increasing the time of exposure to 40 and 60 minutes, ultrasound probe 
increased the ocular tissue temperature. The instilled ascorbic acid on the cornea was protecting the ocular tissues by reducing heat. Therefore, this study recommended considering oral $\beta$-carotene antioxidant in combination with vitamin $\mathrm{C}$ eye drops as a medical tool and personal protective in ultrasound equipment in provide safety.

\section{Conflict of Interest}

The authors declare that they have no competing interests.

\section{References}

[1] Rwei AY, Paris JL, Wang B, et al. (2017) Ultrasound-triggered local anaesthesia. Nat Biomed Eng. 1: 644-653.

[2] Chen Y, Shi Z, Shen Y. (2018) Eye damage due to cosmetic ultrasound treatment: a case report. BMC Ophthalmology. 18: 214.

[3] Eggersdorfer M, Wyss A (2018) Carotenoids in human nutrition and health. Arch Biochem Biophys. Aug 15; 652: 18-26.

[4] Li B, Rognon GT, Mattinson T, Vachali PP, Gorusupudi A, Chang FY (2018) Supplementation with macular carotenoids improves visual performance of transgenic mice. Arch Biochem Biophys. Jul 1; 649: 22-28.

[5] Moschos MM, Dettoraki M, Tsatsos M, Kitsos G, Kalogeropoulos C. (2017) Effect of carotenoids dietary supplementation on macular function in diabetic patients. Eye and Vision. 4: 23.

[6] Giannaccare G, Pellegrini M, Senni C, Bernabei F, Scorcia V, Cicero AFG. (2020) Clinical Applications of Astaxanthin in the Treatment of Ocular Diseases: Emerging Insights. Mar Drugs. 18 (5): 239. Published 2020 May 1.

[7] Berman, J., et al. (2014) Nutritionally important carotenoids as consumer products. Phytochemistry Review, 14: 727-743.

[8] Harasym, J., Oledzki, R. (2014) Effect of fruit and vegetable antioxidants on total antioxidant capacity of blood plasma. Nutrition, 30: 511-517.

[9] McGahan, M. c. (1985) Ascorbic acid levels in aqueous and vitreous humors of the rabbit, Exp. Eye Res. 41: 291-298.

[10] Varma, S. D., and Richards, R. D., (1988) Ascorbic acid and the eye lens, Ophthalmic Res. 20: 164-173.

[11] Vizzarri F, Palazzo M, Bartollino S, et al. (2018) Effects of an antioxidant protective topical formulation on eye exposed to ultraviolet-irradiation: a study in rabbit animal model. Physiol Res. 67 (3): 457-464.

[12] Bartollino, S., Palazzo, M., Semeraro, F. et al., (2020) Effects of an antioxidant protective topical formulation on retinal tissue of UV-exposed rabbits. Int Ophthalmol 40, 925-933.

[13] Nemet AY, Assia EI, Meyerstein D, Meyerstein N, Gedanken A, Topaz M. (2007) Protective effect of freeradical scavengers on corneal endothelial damage in phacoemulsification. J Cataract Refract Surg. 33: 310-315. man: in search of treatment for photoreceptor degeneration. Friedenwald lecture. Invest Ophthalmol Vis Sci.30 (12): 2430-2454.

[15] El Refaei F. M., Karam S. H., Ibrahim I. H., Sallam A. M., Ali M. (2007) Biometrical Histological Study of Rabbits' Eye Following Exposure to Therapeutic Doses of Ultrasound. In: Magjarevic R., Nagel J. H. (eds) World Congress on Medical Physics and Biomedical Engineering 2006. IFMBE Proceedings, vol 14. Springer, Berlin, Heidelberg.

[16] Pastor-Idoate S, Bonshek R, Irion L, et al. (2017) Ultra structural and histopathology findings after pars plana vitrectomy with a new hypersonic vitrector system. Qualitative preliminary assessment. PLoS One. 12 (4).

[17] O'Daly B, Morris E, Gavin G, O'Byrne J, McGuinness G. (2008) High-power low-frequency ultrasound: A review of tissue dissectionand ablation in medicine and surgery. J. Mater. Process. Technol. $200(1 \pm 3), 38 \pm 58$.

[18] Rocha F, Yumi Sugahara L, Leimann FV, et al. (2018) Nanodispersions of beta-carotene: effects on antioxidant enzymes and cytotoxic properties. Food Funct. 9 (7): 3698-3706.

[19] Williams DM, Hägg S, Pedersen NL. Circulating antioxidants and Alzheimer disease prevention: a Mendelian randomization study. Am J Clin Nutr. 2019; 109 (1): 90-98.

[20] Latief U, Ahmad R. $\beta$-carotene inhibits NF- $\kappa \mathrm{B}$ and restrains diethylnitrosamine-induced hepatic inflammation in Wistar rats [published online ahead of print, $2020 \mathrm{Jul}$ 20]. Int J Vitam Nutr Res. 2020; 1-10.

[21] Ham WT, Jr, Mueller HA, Ruffolo JJ, Jr, et al: Solar retinopathy as a function of wavelength: Its significance for protective eyewear. In The Effects of Constant Light on VisuaL Processes, William TP and Baker BN, editors. New York, Plenum Press, 1980, pp. 319-346.

[22] Hatab MR, Giller CA, Clarke GD. Evaluation of cerebral arterial flow with transcranial doppler ultrasound: theoretical development and phantom studies. Ultrasound Med Biol 1997; 23: 1025-31.

[23] Topaz M, Motiei M, Assia E, Meyerstein D, Meyerstein N, Gedanken A: Acoustic cavitation in phacoemulsification: Chemical effects, modes of action and cavitation index. Ultrasound Med Biol 2002; 28: 775-84.

[24] Rubowitz A, Assia EI, Rosner M, Topaz M. Antioxidant protection against corneal damage by free radicals during phacoemulsification. Invest Ophthalmol Vis Sci. 2003; 44: 1866-1870.

[25] Arash RM. High intensity focused ultrasound in ophthalmology: part one, transscleral drug delivery: part two, infrared thermography for scalable acoustic characterization, an application in the manufacture of a glaucoma treatment device. Human health and pathology. Université Claude Bernard-Lyon I, 2014. English.

[26] Abdel-Dayem, Hisham \& Hartzer, Michael \& Williams, George \& Ferrone, Philip. (2017). The Effect of Vitrectomy Infusion Solutions on Postoperative Electroretinography and Retina Histology. BMJ Open Ophthalmology.

[14] Tso MO. (1989) Experiments on visual cells by nature and 